\section{A Modified Total Thigh Flap in the Reconstruction of Decubitus Ulcer}

\author{
Jae Hoon Shin, In Pyo Hong, Chul Gyoo Park, \\ Chan Min Chung \\ Department of Plastic and Reconstructive surgery, National \\ Medical Center, Seoul, Korea
}

\begin{abstract}
Correspondence: In Pyo Hong
245 Eulji-ro, Jung-gu, Seoul 100-799, Korea

Tel: +82-2-2260-7207, Fax: +82-2-2272-7207
\end{abstract}

Department of Plastic and Reconstructive Surgery, National Medical Center,

E-mail:nmcps@unitel.co.kr

No potential conflict of interest relevant to this article was reported.

Received: 20 Mar 2014 • Revised: 4 Jun 2014 • Accepted: 4 Jun 2014 pISSN: 2234-6163・ elSSN: 2234-6171

http://dx.doi.org/10.5999/aps.2014.41.4.440 • Arch Plast Surg 2014:41:440-442

Copyright (C) 2014 The Korean Society of Plastic and Reconstructive Surgeons

This is an Open Access article distributed under the terms of the Creative Commons Attribution Non-Commercial License (http://creativecommons.org/licenses/by-nc/3.0/) which permits unrestricted non-commercial use, distribution, and reproduction in any medium, provided the original work is properly cited.

A total thigh flap may be another treatment option that surgeons may consider when they cannot achieve a coverage of the defect using other treatment modalities in patients with extensive decubitus ulcer

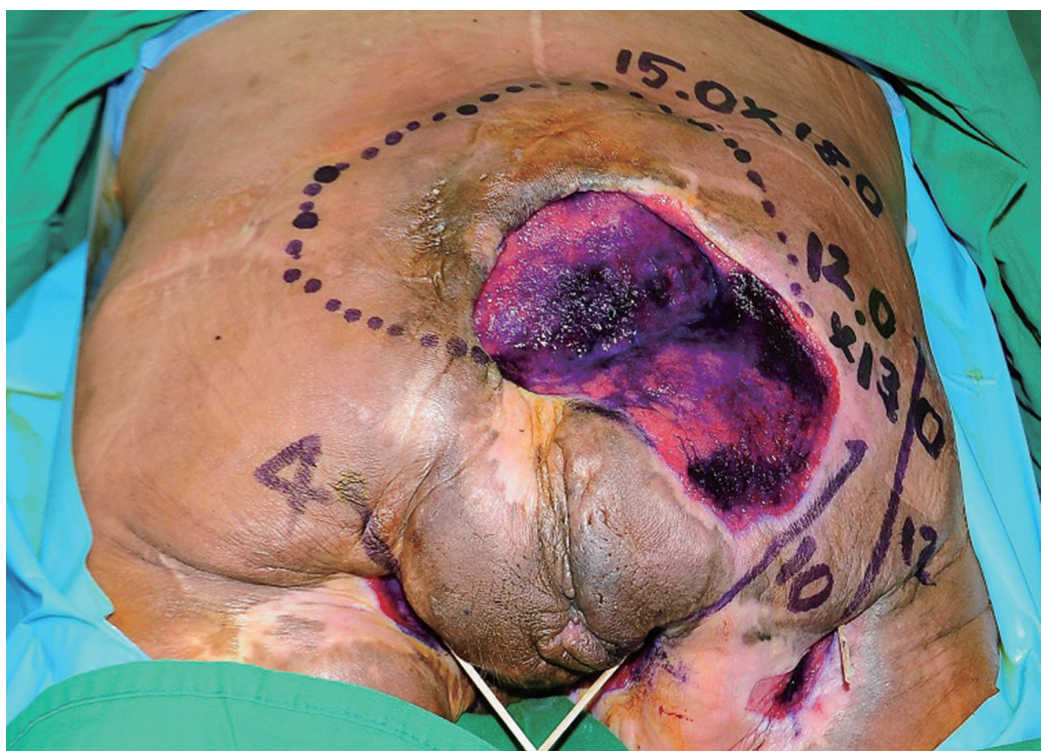

Fig. 1.

The patient had a paraplegia on admission, and had a past history of taking surgery for decubitus ulcer of the sacral and ischial regions several times. In addition, the patient had a deep decubitus ulcer of approximately $15.0 \times 18.0 \mathrm{~cm}$ in the sacral region, $3.0 \times 4.0 \times 12.0$ $\mathrm{cm}$ in the right ischial one and approximately $2.0 \times 3.0 \times 4.0 \mathrm{~cm}$ and $3.0 \times 4.0 \times 10.0 \mathrm{~cm}$ in the left and right subscrotal ones, respectively, where the healthy anal tissue was present as a bridge. Its adjacent area was characterized by the accumulation of scar and granulation tissue due to previous surgeries. concurrently with complications. It has a sufficient vascular supply and is therefore advantageous in providing a sufficient thickness, thus possibly minimizing the recurrence of decubitus ulcer.

We divided the thigh muscle into the anterior and posterior compartment depending on the vascular territory to the desirable shape and size without affecting the healthy tissue. Thus, we restored defect sites to the natural shape.

A 56-year-old man recently sustained grade 4 decubitus ulcer of the sacral, right ischial and bilateral subscrotal regions, accompanied by femoral osteomyelitis. The patient had a spinal cord injury due to the compression fracture of the 11th and 12th thoracic vertebrae and the 1st and 2nd lumbar ones, thus presenting with paraplegia. The patient had a past history of taking surgery for decubitus ulcer of the sacral and ischial regions (Fig. 1).

In the right ischium, the tunnel connected decubitus ulcer to the proximal femur. On X-ray findings, there were multiple bony lesions in the ischium. On magnetic resonance imaging (MRI) scans, there was osteomyelitis accompanied by pathologic fracture in the proximal femur. On bone scan scintigraphy, there were findings that are suggestive of increased uptake and multiple osteomyelitis of the sacrum and ischium at the sites of decubitus ulcer. A bacterial culture test identified such bacterial strains as Pseudomonas aeruginosa and Klebsiella pneumoniae.

The patient refused to take lower extremity amputation, and underwent dissection of the subtrochanteric area involving the right ischium. We therefore elevated semitendinosus and biceps femoris flaps to restore the sites of decubitus ulcer.

From postoperative day 3 on, the patient had wound disruption with yellowish discharge at suture margins, accompanied by gross findings of inflammatory responses in the adjacent tissue accompanied by the partial necrosis of the femur. Therefore, we performed the infection control using daily surgical dressing, AQUACEL hydrofiber Ag (Convatec, Princeton, NJ, USA) and vacuum assisted wound dressing for approximately 15 days. Before the flap procedure, we performed a 3-dimensional (3D) femoral artery computed tomography (CT) to confirm major branches of the deep femoral artery and thereby evaluated the shape and type of preoperative vessels of the descending branch and medial circumflex branch of the lateral circumflex artery [1]. In addition, we also performed colostomy 

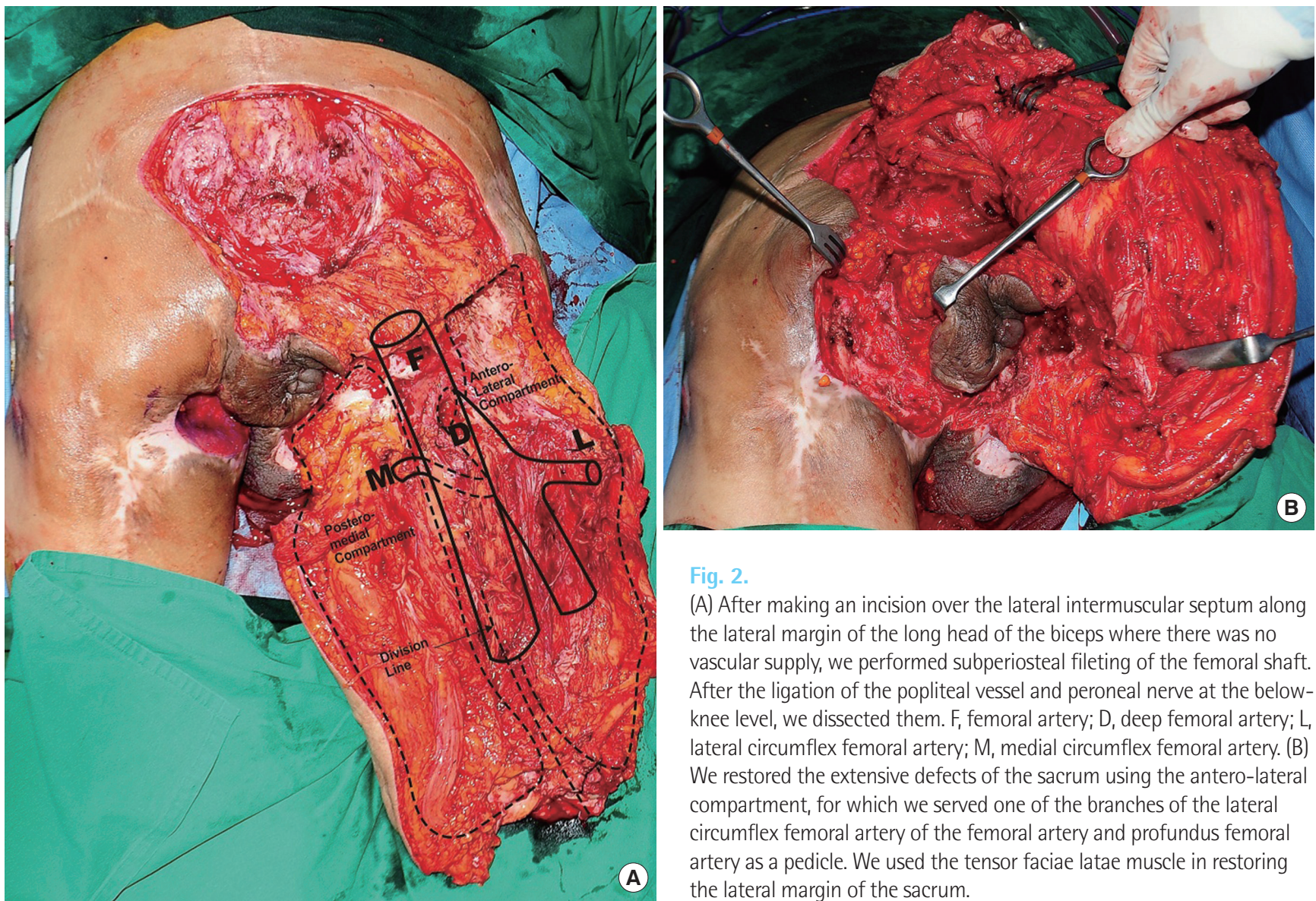

Fig. 2.

(A) After making an incision over the lateral intermuscular septum along the lateral margin of the long head of the biceps where there was no vascular supply, we performed subperiosteal fileting of the femoral shaft. After the ligation of the popliteal vessel and peroneal nerve at the belowknee level, we dissected them. F, femoral artery; $D$, deep femoral artery; $L$, lateral circumflex femoral artery; $\mathrm{M}$, medial circumflex femoral artery. (B) We restored the extensive defects of the sacrum using the antero-lateral compartment, for which we served one of the branches of the lateral circumflex femoral artery of the femoral artery and profundus femoral artery as a pedicle. We used the tensor faciae latae muscle in restoring the lateral margin of the sacrum.

to remove major risk factors of developing infections that may impair the wound healing process. We performed the systemic infection control and daily surgical dressing for approximately one month and 15 days.

Through an incision over the lateral intermuscular septum along the lateral margin of the long head of the biceps, we performed subperiosteal fileting of the femoral shaft not to damage the profunda femoris vessels and then elevated a total thigh flap (Fig. 2A) [2].

For the reconstruction of colostomy, we restored the extensive defects of the sacrum using the anterolateral compartment, for which we served one of the branches of the lateral circumflex femoral artery of the femoral artery and profundus femoral artery as a pedicle. We used the tensor faciae latae muscle in restoring the lateral margin of the sacrum (Fig. 2B). In addition, we also restored the remaining defects of the sacrum by elevating flaps from such muscles as the vastus medialis, rectus femoris, vastus intermedius and vastus lateralis and thereby obtaining a sufficient amount of padding [3].
After simply folding the antero-lateral compartment and then fixed it to the superior sacral region, we tailored the postero-lateral compartment to the shape of the defect sites to cover the right ischial and both perineal defects by serving one of the branches of the medial circumflex femoral artery and the perforating branch of the profundus femoral artery as a pedicle. Then, we also tailored the sartorius muscle from the anterior compartment and the adductor longus muscle from the posterior compartment to the distal part of the upper margin of the anus, for which we pulsated arteries that are responsible for blood supply in each muscle.

With the isolation of the gracilis, adductor magnus and adductor longus muscle using an electrocautery, we folded them in the dead space around the anus and thereby obtained the padding effect. Then, we restored the subscrotal margin using the biceps femoris muscle [4]. We finally adjusted the thickness of the flap using the semimembranosus and semitendinosus muscle through a partial resection of the fascia and adipose tissue except for the vascular pedicle (Fig. 3A).

On postoperative day 10 , the patient developed a 

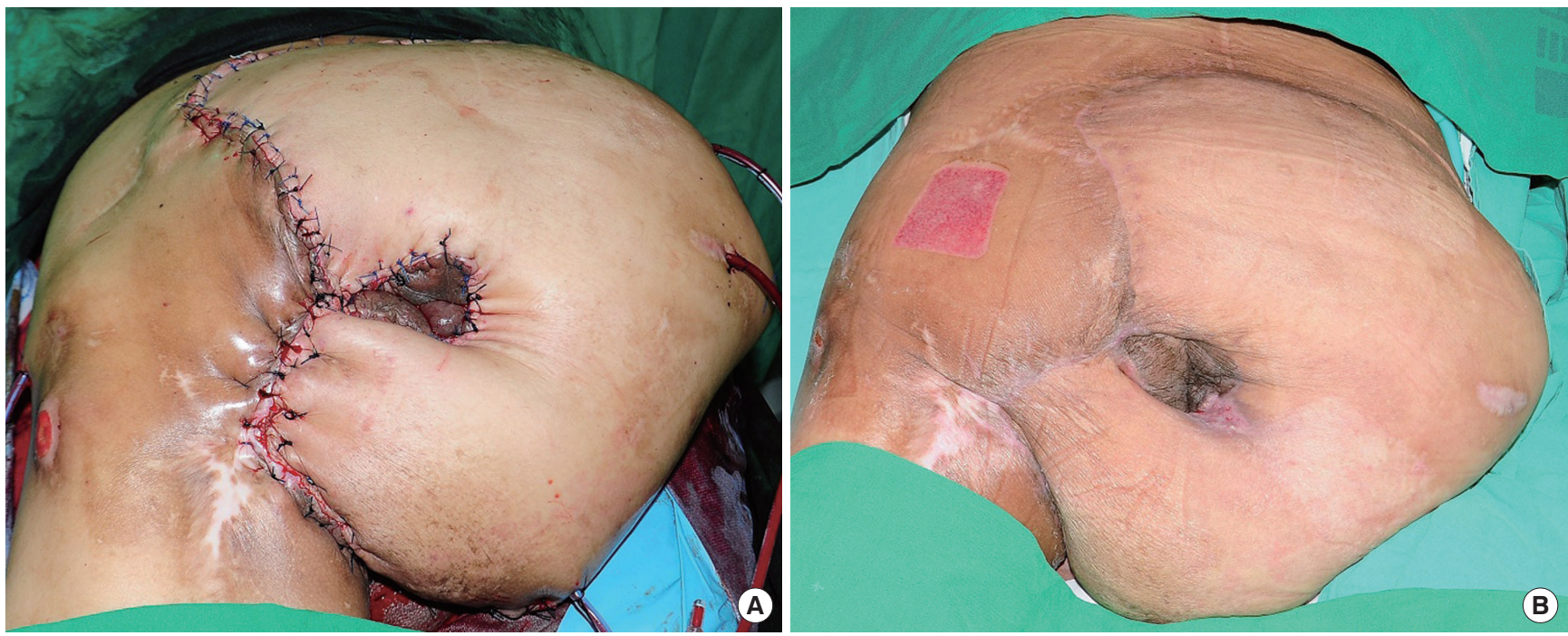

Fig. 3.

(A) We isolated the gracilis, adductor magnus and adductor longus muscle using an electrocautery and then folded them in the dead space around the anus, thus obtaining a padding effect. After the restoration of the subscrotal margin using the biceps femoris muscle with the arc of rotation and a high relaxation ability, we used the semimembranosus and semitendinosus muscle for a partial resection of the fascia and adipose tissue except for the vascular pedicle to the shape of the recipient site, thus adjusting the thickness of the flap. (B) On postoperative day 10, after the removal of a drain, the patient developed a necrosis on the medial side of the suture site from the adjacent flap. But this was easily resolved using a resection and a splitthickness skin graft. One month later, the patient was discharged from us.

necrosis on the medial side of the suture site from the adjacent flap. But this was easily resolved using a resection and a split-thickness skin graft. One month later, the patient was discharged from us (Fig. 3B).

In 1956, Georgiade et al. [5] first attempted to use a total thigh flap in the treatment of patients with multiple decubitus ulcer who concurrently had complications. Since then, several authors have also used it in a same series of patients. According to most of the previous reports in this series, however, it is recommended that the defect sites be restored by elevating a total thigh flap and then simply folding a muscle flap. But its applicability is limited to patients with small decubitus ulcer or those whose lesions are limited to the ipsilateral side. We assumed that it would be difficult to make a coverage of the contralateral defects without splitting bulky thigh muscles in patients with extensive lesions. We therefore split the thigh muscles based on the vascular territory and preoperatively performed a 3D CT of the femoral artery considering the possibility of anatomical variations of the major branches of the femoral artery. Thus, we could increase the arc of rotation of the flap and thereby could achieve a coverage of the defect with the elevation of the flap by splitting the thigh muscles even to the contralateral side as well as the ipsilateral one without causing tension or twisting of the thigh muscles.

\section{References}

1. Valdatta L, Tuinder S, Buoro M, et al. Lateral circumflex femoral arterial system and perforators of the anterolateral thigh flap: an anatomic study. Ann Plast Surg 2002;49:145-50.

2. Cheng PT, Adams BM, Chunilal A. Modified total thigh musculocutaneous flap: 'operation of last resort' for massive pressure ulcers. J Plast Reconstr Aesthet Surg 2014;67:260-3.

3. Acarturk TO. Treatment of large ischial ulcers communicating with the hip joint with proximal femoral resection and reconstruction with a combined vastus lateralis, vastus intermedius and rectus femoris musculocutaneous flap. J Plast Reconstr Aesthet Surg 2009;62:1497-502.

4. Friedman JD, Reece GR, Eldor L. The utility of the posterior thigh flap for complex pelvic and perineal reconstruction. Plast Reconstr Surg 2010;126:146-55.

5. Georgiade N, Pickrell K, Maguire C. Total thigh flaps for extensive decubitus ulcers. Plast Reconstr Surg (1946) 1956;17:220-5. 